\title{
Role of Brain Natriuretic Peptide in the Prediction of Early Postoperative Seizures Following Surgery for Traumatic Acute Subdural Hematoma: A Prospective Study
}

\author{
Mehdi Chihi (D) - Marvin Darkwah Oppong · Carlos M. Quesada · Thiemo Florin Dinger • Oliver Gembruch • \\ Daniela Pierscianek • Yahya Ahmadipour · Anne-Kathrin Uerschels · Karsten H. Wrede $\cdot$ Ulrich Sure • \\ Ramazan Jabbarli
}

Received: May 23, 2021 / Accepted: July 21, 2021 / Published online: August 3, 2021

(C) The Author(s) 2021

\section{ABSTRACT}

Introduction: Brain natriuretic peptide (BNP) is a reliable biomarker in the acute phase of traumatic brain injury. However, the relationship between BNP and traumatic acute subdural hematoma (aSDH) has not yet been addressed. This study aimed to analyze BNP levels on admission in surgically treated patients and assess their relationship with early postoperative seizures (EPS) and functional outcomes.

Methods: Patients with unilateral traumatic aSDH who were surgically treated in our department between July 2017 and May 2020 were included in the study. BNP was preoperatively measured. Patients' neurologic condition, radiographic variables on initial cranial computed tomography, sodium serum levels on

Supplementary Information The online version contains supplementary material available at https:// doi.org/10.1007/s40120-021-00269-w.

M. Chihi $(\bowtie) \cdot$ M. Darkwah Oppong ·

T. F. Dinger · O. Gembruch · D. Pierscianek ·

Y. Ahmadipour · A.-K. Uerschels · K. H. Wrede .

U. Sure · R. Jabbarli

Department of Neurosurgery and Spine Surgery, University Hospital Essen, University of DuisburgEssen, Hufelandstrasse 55, 45147 Essen, Germany e-mail: mehdi.chihi@uk-essen.de

C. M. Quesada

Department of Neurology, Center of Epileptology, University Hospital Essen, University of DuisburgEssen, Essen, Germany admission, and occurrence of EPS were prospectively assessed. Functional outcome was assessed using the modified Rankin Scale (mRS) at discharge and follow-up (at 2-3 months). A poor outcome was defined by a mRS score $>3$. Results: EPS occurred in 20 (19.6\%) of 102 surgically treated patients in the final cohort on the median day 3. A significant association between EPS and a poor Glasgow Coma Scale score at the 7th postoperative day was found, which in turn independently predicted a poor functional outcome at discharge and follow-up. Nonetheless, EPS were not associated with poor functional outcomes. The multivariate analysis revealed $\quad \mathrm{BNP}>95.4 \mathrm{pg} / \mathrm{ml} \quad(\mathrm{aOR}=5.7$, $p=0.003)$, sodium $<137.5 \mathrm{mmol} / \mathrm{l}(\mathrm{aOR}=4.6$, $p=0.009)$, and left-sided aSDH $(\mathrm{aOR}=4.4$, $p=0.020)$ as independent predictors of EPS.

Conclusion: In the early postoperative phase of traumatic aSDH, EPS were associated with worse neurologic conditions, which in turn independently predicted poor outcomes at discharge and follow-up. Although several EPS risk factors have already been elucidated, this study presents BNP as a novel reliable predictor of EPS. Further larger studies are needed to determine whether a more precise estimate of EPS risk using BNP levels can be reached.

Keywords: Brain natriuretic peptide; Early postoperative seizures; Posttraumatic seizures; Acute subdural hematoma; Biomarker; Antiepileptic drugs 


\section{Key Summary Points}

\section{Why carry out this study?}

Early postoperative seizures (EPS) following surgery for traumatic acute subdural hematoma are considered a leading prognostic factor. Some recent studies estimated their incidence between 24 and 36\%, which is significantly higher than that of posttraumatic seizures (only 5-7\%). Nevertheless, the use of prophylactic antiepileptic drugs in the acute phase to reduce the risk of early seizures remains controversial, and there are to date no clinical evidence compared to that in severe traumatic brain injury, by which phenytoin is recommended in the first week after trauma (American Academy of Neurology, Class I evidence, Level A recommendation).

In this study, the incidence of early postoperative seizures in the setting of traumatic acute subdural hematoma was assessed, and the role of the biomarker "brain natriuretic peptide" in the prediction of early postoperative seizures was investigated.

\section{What was learned from the study?}

Early postoperative seizures are a common complication in the early postoperative phase of traumatic acute subdural hematoma, as their occurrence was associated with worse neurologic conditions on the 7 th postoperative day, which in turn predicted poor functional outcomes at discharge and follow-up.

However, EPS were not directly associated with poor outcomes.

Brain natriuretic peptide serum level on admission is a novel reliable predictor of early postoperative seizures following surgery for traumatic acute subdural hematoma. The study predictive model could stratify the risk of experiencing early postoperative seizures in three categories (low, intermediate, and high risk) and may help neurosurgeons and neurologists in further evaluation of that risk. Furthermore, our study results may serve as paradigm for future research in the field of early postoperative seizures.

\section{INTRODUCTION}

Traumatic acute subdural hematoma (aSDH) is a major clinical entity in traumatic brain injury (TBI) that is often complicated by co-existing intracranial lesions, including an extra-axial space-occupying hematoma, parenchymal contusions, brain edema, and a variety of diffuse injuries $[8,20,30,43]$. ASDH is a common disease that is increasing in prevalence and costs, accounting for about 90,000 hospitalizations annually in the USA [11]. It is considered the most frequent extra-axial hemorrhage requiring emergent neurosurgical evacuation and immediate intensive care [11]. The identification of prognostic factors such as patient age, clinical status, signs of cerebral herniation, hematoma size, time until treatment, and postraumatic seizures $[30,33,41]$ is therefore highly necessary in the clinical setting for predicting patients' clinical course and outcome [8].

Early posttraumatic seizures (PTS), occurring within the first week after head trauma, are a common complication in patients with traumatic aSDH $[16,38]$. Early PTS that occurred after hematoma evacuation-or early postoperative seizures (EPS) - are to date not described enough $[30,43]$. Indeed, there is a paucity of research investigating the relationship between EPS and aSDH, and this association has not yet been sufficiently characterized. To date, a few studies have addressed the incidence and impact of EPS on functional outcomes in patients with aSDH. Their incidence was reported in only four studies [16, 30, 38, 43] and ranged between 24 and 36\%, significantly higher than the general incidence of early PTS (estimated between 5 and 7\%) [39, 46]. Additionally, EPS in aSDH patients delays favorable recovery and is independently associated with worse functional outcome at discharge $[27,30,32,33,44]$. Other authors found that EPS worsened both early and late functional outcome and was significantly associated with higher mortality rate $[8,43]$. Therefore, early identification of patients at high risk of EPS in clinical practice could be helpful for counseling patients and their relatives regarding the early functional outcome. 
Recent studies have reported an increase in plasma brain natriuretic peptide (BNP) in the acute phase of TBI, which is related to the elevation of the intracranial pressure (ICP) [21, 35, 45], hyponatremia [45], trauma severity [45], and clinical outcomes [35]. BNP is a vasorelaxant peptide [1] mainly expressed in the ventricular myocardium [25] that reaches the hypothalamus through the subfornical organ [1]. BNP plays a central role in the modulation of cardiovascular homeostasis as well as the regulation of hypothalamic-pituitary-adrenal axis function [29] and is considered a reliable biomarker in TBI. Nevertheless, the relationship between BNP and traumatic aSDH, especially the risk of EPS following hematoma evacuation, has not yet been addressed.

In light of these knowledge gaps [21, 35, 45], we aimed in this study to investigate the clinical implications of elevated BNP for the prediction of EPS and functional outcomes in patients with traumatic aSDH.

\section{METHODS}

\section{Study Population}

Between July 2017 and May 2020, 163 patients aged $\geq 18$ years with isolated unilateral aSDH that were admitted at our institution were evaluated for eligibility for this study. Patients with a history of chronic heart or renal failure, brain surgery, intracranial tumor, aneurysmatic subarachnoid hemorrhage, or interhemispheric, bilateral, or non-traumatic aSDH were excluded. Of the 136 eligible patients, all individuals who were not surgically treated were not included in the final cohort $(N=102)$. The study flowchart summarizes the main exclusion criteria in detail (Fig. 1).

\section{Ethical Standards}

This prospective study was performed in accordance with the ethical standards of the institutional research committee and the Code of Ethics of the World Medical Association (Declaration of Helsinki). The Institutional Review
Board approved the study protocol (Medical Faculty, University of Duisburg-Essen, Registration number: 15-6632-BO). Written informed consent was obtained from all participants or their next of kin in case of altered state of consciousness.

\section{Routine Clinical Care and Treatment Protocol of Traumatic aSDH}

Pre-existing antiplatelet/anticoagulant therapy (AAT) was discontinued upon hospital admission and re-established 3 weeks after surgery in case of complete resolution of the hematoma. Patients with a Glasgow Coma Scale (GCS) score $<15$ or the presence of a midline shift (MLS) received an immediate hematoma evacuation under general anesthesia with a decompressive craniectomy or craniotomy, depending on presence of brain edema. Prior to surgery, a single dose of cefazolin was administrated prophylactically. Postoperatively, patients spent at least $24 \mathrm{~h}$ in the intensive care unit (ICU) and remained on bed rest. A postoperative CCT was performed on the same day in all cases to rule out a re-bleeding. Patients with a GCS score of 15 without MLS were neurologically monitored for $24 \mathrm{~h}$ in the ICU. A control CCT was performed $6 \mathrm{~h}$ after the first imaging or in response to neurologic deterioration. At this stage, patients that were not operated were secondarily excluded from the study. Patients with initially non-reactive and wide pupils $>2 \mathrm{~h}$ were not operated because of worse prognosis. The Glasgow Coma Scale (GCS) score was prospectively assessed on admission and on the 7 th postoperative day. The occurrence and recurrence of EPS, seizure type, and use of antiepileptic drugs (AEDs) were documented. In case of self-limited EPS, a repeated cranial computed tomography (CCT) was performed to rule out a re-bleeding or cerebral infarction, and venous blood was sampled to rule out electrolyte disorders including sodium, potassium, chloride, calcium, magnesium, and phosphate concentrations. If EPS was prolonged $>30-60$ s, a bolus of $1 \mathrm{mg}$ lorazepam was intravenously administrated to stop the seizure, and then CCT was performed. Levetiracetam was used as the 
Recruitment period

July 2017 - May 2020



Fig. 1 Study flowchart

first-choice AED for treatment. In case of EPS recurrence, the antiepileptic therapy was adjusted/escalated in cooperation with a neurologist. In case of any unexpected neurologic deterioration in absence of previous seizures, a CCT was done, and in case of the absence of an organic cause (re-bleeding/cerebral infarction), an electroencephalogram (EEG) was performed to diagnose/rule out EPS. All EEGs were read by our epileptologist (third author, C.M.Q) and were considered positive if epileptiform or ictal activity was noted in the read. In our institution, AED prophylaxis in asymptomatic patients, or in patients who had had only one self-limited seizure before surgical treatment, was not used. All patients with EPS had an additional follow-up after hospital discharge by a neurologist.

\section{BNP Sampling}

BNP was measured from venous blood samples collected at admission. An electrochemiluminescence immunoassay was performed (Siemens, ADVIA Centauer ${ }^{\circledR}$, USA, manufacturer range $2-5000 \mathrm{pg} / \mathrm{ml}$ ).

\section{Patient Data Collection}

The following medical records were prospectively assessed by the first author (M.C.) using a 
study questionnaire on admission to examine certain data points: age, sex, suspected seizure before head trauma, preoperative seizure, dementia, cardiac history (atrial fibrillation, coronary heart disease), AAT, alcohol abuse before trauma (when ethanol serum level on admission was $>100 \mathrm{mg} / \mathrm{dl}$ ), neurologic condition on admission using the GCS score, and sodium serum concentration on admission. Exact time of head trauma was assessed through anamnesis or after consulting emergency doctor protocols. Furthermore, aSDH location, operative technique, time until treatment (difference between time of incision and time of head trauma in minutes), and $\mathrm{ABO}$ blood type were documented. Additionally, hematoma volumes and MLS were assessed and calculated using iPlan Software (BrainLab, Munich, Germany) on initial CCT. Moreover, brain edema was assessed using the Subarachnoid Hemorrhage Early Brain Edema Score (SEBES), and the presence of brain contusions was documented. Along patients' period of hospitalization, neurologic condition on the 7th postoperative day, inhospital mortality, and postoperative complications such as EPS, cerebral infarction, rebleeding, long-term ventilation ( $>7$ days), acute renal failure, myocardial infarction, and cardiac arrest were assessed. Before being digitized, the data were added to the study questionnaire. Functional status of the patients was also assessed and documented at discharge and last follow-up using a simplified modified Rankin Scale (mRS) questionnaire [4]. A mRS score $>3$ was considered a poor outcome.

\section{Definition of EPS}

According to previous literature [30, 38, 39, 44, 46], EPS were defined as acute symptomatic generalized or focal onset seizures occurring in the postoperative period within 7 days of head trauma. These included tonicclonic seizures, automatisms, or repeated automatic movements like clapping, rubbing of hands, or chewing, with or without impairment of awareness, and seizures with no manifestation apart from brief impairment of awareness.

\section{Follow-up}

Patients visited our outpatient clinic for followup after the rehabilitation period (2-3 months after discharge), and their functional status was assessed.

\section{Statistical Analyses}

All analyses were performed using SPSS version 25 (IBM, Chicago, IL). Categorical data are presented as frequencies and percentages. Nonnormally distributed continuous variables are expressed as the median and the interquartile range (IQR). The Mann-Whitney $U$ test was used to assess statistical associations between nonnormally distributed continuous and dichotomous variables. Associations between categorical variables were determined using the chisquare test and Fisher's exact test (when the expected cell frequency was $<5$ ). Associations between ordinal variables such as GCS/mRS scores/SEBES and dichotomous variables were determined using the Kruskal-Wallis $H$ test. For associations between ordinal and continuous variables, Spearman's correlation was performed. The Cochran-Armitage test of trend was used to assess the association between a dependent dichotomous and an independent ordinal variable. BNP was used as a continuous variable in the univariate analyses. The mRS score was dichotomized ( $\leq 3$ vs. $>3$ ) only when predicting outcome; otherwise, it was used as an ordinal independent variable. The factors associated with the occurrence of EPS in the univariate analyses with a $p$ value $<0.01$ were implemented in the multivariate analyses. Receiver-operating characteristic (ROC) curve analyses were conducted to determine cutoff values of BNP and sodium concentrations and assessment of diagnostic accuracy of prediction models including the BNP and sodium cutoff values. Hierarchical binomial logistic regression was performed first using BNP and sodium levels as continuous variables and then using their corresponding cutoff values to determine predictors of EPS. For each predictor, an adjusted odds ratio (aOR) and 95\% confidence intervals $(95 \% \mathrm{CI})$ were calculated. A prediction 
model based on the results of the multivariate analysis was computed. To assess eventual associations between EPS/GCS on 7th postoperative day and outcomes, we conducted a sensitivity analysis in which patients who died within 7 days of head trauma were excluded. After dichotomizing the outcome variable (mRS score $\leq 3$ vs. $>3$ ), a hierarchical binomial regression analysis was used to determine predictors of postoperative outcome at discharge and follow-up. $P$ values $<0.05$ in two-sided testing were considered statistically significant.

\section{RESULTS}

\section{Baseline and Radiographic Characteristics}

The selection process of the patients eligible for this study is presented in Fig. 1. Accordingly, 102 patients (median age 74 years, male/female 1.48/1) met the inclusion criteria and were included in the study. Of the participants, 17 had atrial fibrillation (16.7\%), 15 had coronary heart disease $(14.7 \%)$, and 41 received AAT $(40.2 \%)$. Nine patients $(8.8 \%)$ were diagnosed with dementia prior to admission and 20 (19.6\%) under alcohol abuse. At admission, 49 patients $(48 \%)$ had a GCS score $\leq 8$. In-hospital mortality rate was $33.3 \%(n=34)$, and 58 of 68 survivors could be followed up at 2-3 months (85.3\%). Severe brain edema was observed in 43 patients $(42.2 \%)$, cerebral herniation in 8 patients $(7.8 \%)$, and parenchymal contusions in 26 patients $(25.5 \%)$ (Table 1$)$. Further characteristics of deceased patients are summarized in Table I (Supplementary Material). Withdrawal of care was performed in accordance with the patient's decree when the patients postoperatively did not experience any neurologic improvement in the absence of any clinical or electroencephalographic signs of seizures and despite the probatory use of AEDs.

\section{BNP Characteristics}

BNP was significantly higher in females, older patients, individuals with cardiac history (i.e., atrial fibrillation, coronary heart disease), and those on AAT. EPS, recurrent EPS, and postoperative myocardial events (i.e., myocardial infarction, cardiac arrest) were statistically significantly associated with higher BNP. The mRS score at discharge was not associated with BNP; however, a statistically non-significant trend between higher BNP levels and poor mRS at follow-up was observed (Table II, Supplementary Material).

\section{Characteristics and Predictors of EPS}

EPS (median: 3 days, 95\% CI [2.3-3.7]) occurred in 20 patients $(19.6 \%)$ and were recurrent in 14 (70\%) of cases. EPS characteristics are summarized in Table III (Supplementary Material). In the univariate analyses, the occurrence of EPS was associated with female sex, advanced age, leftsided aSDH, higher BNP, lower sodium, and longer time until treatment. Furthermore, patients with a worse GCS score at the 7th postoperative day had significantly higher rates of EPS (Table 2).

After adjusting for age, sex, and time until treatment, the hierarchical logistic binomial regression revealed that higher BNP levels, lower sodium concentrations, and left-sided aSDH are independent predictors of EPS (Table 3). The model could explain 35.8\% (Nagelkerke $R^{2}$ ) of the variance in EPS and correctly classified $85.3 \%$ of cases. In the interests of simplicity for use in clinical practice, ROC curve analysis for the prediction of EPS was performed to determine the cutoff values of BNP (Fig. 2) and sodium concentrations which were $\quad 95.4 \mathrm{pg} / \mathrm{ml} \quad(\mathrm{AUC}=0.72 \quad[95 \% \quad \mathrm{CI}$ 0.58-0.85], sensitivity $=70 \%, \quad$ specificity $=70.7 \%)$ and $137.5 \mathrm{mmol} / \mathrm{l}(\mathrm{AUC}=0.70$ $[95 \%$ CI $0.54-0.84]$, sensitivity $=78 \%$, specificity $=60 \%$ ). The hierarchical logistic binomial regression considering both cutoff values revealed that $\mathrm{BNP}>95.4 \mathrm{pg} / \mathrm{ml} \quad(\mathrm{aOR}=5.7)$, sodium $<137.5 \mathrm{mmol} / \mathrm{l}(\mathrm{aOR}=4.6)$, and leftsided aSDH $(\mathrm{aOR}=4.4)$ were independent predictors of EPS (Table 3). According to Hosmer et al. [15], the model's discriminative capacity was determined by a ROC curve analysis and was excellent with an AUC of 0.81 (95\% CI 0.69-0.92). The logistic regression equation was as follows: 
Table 1 Patients' baseline and radiographic characteristics (complete cohort)

\begin{tabular}{|c|c|c|}
\hline Parameters & $N / n$ & $\%$ \\
\hline Age $($ median $=74$ years, $\mathrm{IQR}=26$ years $)$ & 102 & 100 \\
\hline Sex (male/female: $1.48 / 1$ ) & 102 & 100 \\
\hline Female & 41 & 40.2 \\
\hline Male & 61 & 59.8 \\
\hline Suspected seizure before head trauma & 2 & 1.9 \\
\hline Preoperative seizure & 10 & 9.8 \\
\hline Cardiac history & 27 & 26.5 \\
\hline Atrial fibrillation & 17 & 16.7 \\
\hline Coronary heart disease & 15 & 14.7 \\
\hline Antiplatelet/anticoagulant therapy & 41 & 40.2 \\
\hline Dementia & 9 & 8.8 \\
\hline GCS score at admission $\leq 8$ & 49 & 48 \\
\hline Alcohol abuse before trauma (ethanol serum level $>100 \mathrm{mg} / \mathrm{dl}$ ) & 20 & 19.6 \\
\hline \multicolumn{3}{|l|}{ Pupils at admission } \\
\hline Narrow pupils with normal reaction to light & 75 & 73.5 \\
\hline Anisocoria and non-reactive ipsilateral pupil & 18 & 17.6 \\
\hline Wide and non-reactive pupils & 9 & 8.8 \\
\hline \multicolumn{3}{|l|}{ ABO blood type } \\
\hline $\mathrm{O}$ & 35 & 34.4 \\
\hline A & 46 & 45.1 \\
\hline B & 13 & 12.7 \\
\hline $\mathrm{AB}$ & 8 & 7.8 \\
\hline \multicolumn{3}{|l|}{ Operative technique } \\
\hline Craniotomy & 29 & 28.4 \\
\hline Craniectomy & 73 & 71.6 \\
\hline Time until treatment $($ median $=345 \mathrm{~min}, \mathrm{IQR}=663 \mathrm{~min})$ & 102 & 100 \\
\hline In-hospital mortality & 34 & 33.3 \\
\hline $\mathrm{mRS}$ score at discharge $>3$ & 85 & 84.6 \\
\hline $\mathrm{mRS}$ score at last follow-up ${ }^{\mathrm{a}}>3$ & 30 & 51.7 \\
\hline Parenchymal contusions on initial CCT & 26 & 25.5 \\
\hline Severe brain edema $($ SEBES $>2)$ & 43 & 42.2 \\
\hline Cerebral herniation & 8 & 7.8 \\
\hline Hematoma volume $($ median $=59.9 \mathrm{ml}, \mathrm{IQR}=69.9 \mathrm{ml})$ & 102 & 100 \\
\hline
\end{tabular}


Table 1 continued

\begin{tabular}{lll}
\hline Parameters & $\boldsymbol{N} / \boldsymbol{n}$ & $\%$ \\
\hline Midline shift $($ median $=5.4 \mathrm{~mm}, \mathrm{IQR}=8.2 \mathrm{~mm})$ & 102 & 100 \\
Sodium serum level on admission $($ median $=139 \mathrm{mmol} / \mathrm{l}, \mathrm{IQR}=6 \mathrm{mmol} / \mathrm{l})$ & 102 & 100 \\
\hline
\end{tabular}

$C C T$ cranial computed tomography, $C I$ confidence intervals, $m R S$ modified Rankin Scale, GCS Glasgow Coma Scale, IQR interquartile range, SEBES subdural hemorrhage early brain edema score

a 58 (followed up) of 68 patients (survived until discharge)

$$
\begin{aligned}
\operatorname{Logit}(p)= & -3.775+1.755 \times \mathrm{BNP}+1.525 \\
& \times \text { sodium }+1.473 \times \text { aSDH location }
\end{aligned}
$$

\section{Predictive Model}

A predictive model was designed using BNP and sodium levels with their cutoff values and aSDH location. One point per variable was attributed in case of $\mathrm{BNP}>95.4 \mathrm{pg} / \mathrm{ml}$, sodium $<137.5 \mathrm{mmol} / \mathrm{l}$, and left-sided aSDH. A minimum score of 0 indicates "low risk of EPS" and a maximum score of 3 indicates "high risk of EPS." The model's discriminative capacity was excellent [15], with an AUC of 0.81 (95\% CI 0.69-0.92) (Fig. 3). A score $\geq 2$ (sensitivity: 70\%, specificity: $76.8 \%$ ) was determined as the best threshold to discriminate the risk of experiencing EPS.

\section{Risk Stratification}

Compared to a score of 0 ("low risk"), there was an exponential increase in the risk of developing EPS with a score of 1 ("intermediate risk") or a score of $2 / 3$ ("high risk") $(\mathrm{aOR}=2.9$ [95\% CI $0.316-26.141] \quad$ vs. $\quad$ aOR $=16.9 \quad[95 \% \quad \mathrm{CI}$ 2.039-140.880], respectively) (Fig. 4 and Table 4). Furthermore, the Cochran-Armitage test of trend showed a statistically significant increase in the occurrence rate of EPS with increasing score (Table IV, Supplementary Material).

\section{Sensitivity Analysis}

All statistical analyses were performed again after excluding patients who died within the first 7 days of head trauma. The statistical results were unchanged. Tables V, VI, VIII, IX, and $\mathrm{X}$ (Supplemental Material) summarize in detail these findings.

\section{Postoperative Functional Outcomes}

A poor functional outcome at discharge was significantly associated with female sex, larger hematoma volumes and MLS, advanced age, and higher BNP levels. A poor functional outcome at follow-up was significantly associated with the presence of cardiac history, CHD, larger hematoma volumes and MLS, advanced age, higher BNP levels, and worse GCS scores at admission. Neither EPS nor recurrent EPS were significantly associated with functional outcomes (Table VII, Supplementary Material). The hierarchical binomial regression analysis showed that a poor GCS at admission, advanced age, and larger hematoma volumes are independent predictors of poor functional outcome at discharge and follow-up. In the sensitivity analysis, advanced age and poor GCS score at the 7 th postoperative day were independent predictors of poor functional outcome at discharge, whereas advanced age and poor GCS scores at admission and at the 7 th postoperative day were independent predictors of poor functional outcome at follow-up (Table IX and X, Supplementary Material).

\section{DISCUSSION}

This study is the first to investigate the clinical implications of BNP in surgically treated patients following traumatic aSDH. We found that EPS were associated with a poor neurologic condition of the patients on the 7th 
Table 2 Characteristics of early postoperative seizures (complete cohort)

\section{Early postoperative seizures (univariate analyses)}

\begin{tabular}{|c|c|c|c|c|c|c|}
\hline Parameters (chi-square test) & $n(\%)[\mathrm{EPS}]$ & $n(\%)[$ no EP & & OR & 95\% CI for OR & $p$ value \\
\hline Suspected seizure before head trauma & $0(0)$ & $2(2.5)$ & & - & - & 1.000 \\
\hline Preoperative seizure & $2(10)$ & $8(9.8)$ & & - & - & 1.000 \\
\hline Sex (female) & $14(70)$ & $27(32.9)$ & & 4.8 & $1.64-13.74$ & $0.004^{*}$ \\
\hline ABO blood type (A) & $12(60)$ & $34(43)$ & & - & - & 0.214 \\
\hline Subdural hematoma location (left) & $15(75)$ & $37(45.1)$ & & 3.6 & $1.21-10.98$ & $0.024^{*}$ \\
\hline Alcohol abuse before trauma & $1(5)$ & $19(23.2)$ & & - & - & 0.112 \\
\hline Dementia & $4(20)$ & $5(6.1)$ & & - & - & 0.071 \\
\hline Cardiac history & $8(40)$ & $19(23.2)$ & & - & - & 0.159 \\
\hline Atrial fibrillation & $6(30)$ & $11(13.4)$ & & - & - & 0.095 \\
\hline Coronary heart disease & $3(15)$ & $12(14.6)$ & & - & - & 1.000 \\
\hline Antiaggregant/anticoagulant therapy & $10(50)$ & $31(37.8)$ & & - & - & 0.446 \\
\hline In-hospital mortality & $7(35)$ & $27(32.9)$ & & - & - & 1.000 \\
\hline Operative technique (craniotomy) & $9(45)$ & $20(24.4)$ & & - & - & 0.096 \\
\hline Parenchymal contusions on initial CCT & $6(30)$ & $20(24.4)$ & & - & - & 0.775 \\
\hline Cerebral herniation & $1(5)$ & $7(8.5)$ & & - & - & 0.697 \\
\hline Parameters (Kruskal-Wallis H test) & - & \multicolumn{2}{|l|}{ OR } & \multicolumn{2}{|c|}{$95 \%$ CI for mean } & $p$ value \\
\hline GCS score at admission & - & - & & \multicolumn{2}{|l|}{-} & 0.089 \\
\hline Brain edema (using SEBES) & - & - & & \multicolumn{2}{|l|}{-} & 0.072 \\
\hline Parameters (Mann-Whitney $U$ test) & Median & IQR & OR & & $\% \mathrm{CI}$ for mean & $p$ value \\
\hline Age (years) & 74 & 26 & - & & $.0-81.4$ & $0.014^{*}$ \\
\hline BNP serum level on admission $(\mathrm{pg} / \mathrm{ml})$ & 55.9 & 134.4 & - & & $8.4-409.8$ & $0.002^{*}$ \\
\hline Hematoma volume (ml) & 59.9 & 69.9 & - & & $.70-80.71$ & 0.111 \\
\hline Midline shift (mm) & 5.4 & 8.2 & - & & $36-6.46$ & 0.520 \\
\hline Sodium serum level on admission $(\mathrm{mmol} / \mathrm{l})$ & 139 & 6 & - & & $2.0-138.5$ & $0.008^{*}$ \\
\hline Time until treatment $(\mathrm{min})$ & 345 & 663 & - & & $5-4099.7$ & $0.012^{*}$ \\
\hline
\end{tabular}

$B N P$ brain natriuretic peptide, $C C T$ cranial computed tomography, $m R S$ modified Rankin Scale, GCS Glasgow Coma Scale, $S D H$ subdural hematoma, SEBES subdural hemorrhage early brain edema score

* $p$ value significant

postoperative day, which in turn predicted a poor functional outcome at discharge and follow-up. Nonetheless, EPS were not associated with a poor functional outcome at discharge and follow-up. In addition, higher BNP levels $(>95.4 \mathrm{pg} / \mathrm{ml})$, lower sodium concentrations 
Table 3 Predictors and prediction model of early postoperative seizures (complete cohort). Minimum score 0 (less likely to meet EPS); maximum score 3 (most likely to meet EPS)

Early postoperative seizures $(N=102)$ multivariate analysis and prediction model

\begin{tabular}{|c|c|c|c|c|}
\hline Predictors & $B$ & 95\% CI & $p$ value & Wald \\
\hline BNP serum level on admission & 0.006 & $1.002-1.010$ & $0.003^{*}$ & 9.131 \\
\hline Sodium serum level on admission & -0.122 & $0.795-0.985$ & $0.025^{*}$ & 5.018 \\
\hline SDH location (left) & 1.379 & $1.116-14.142$ & $0.033^{*}$ & 4.534 \\
\hline Age & - & - & 0.151 & - \\
\hline Sex (female) & - & - & 0.128 & - \\
\hline Time until treatment & - & - & 0.430 & - \\
\hline Dementia & - & - & 0.104 & - \\
\hline Atrial fibrillation & - & - & 0.527 & - \\
\hline Operative technique (craniotomy) & - & - & 0.097 & - \\
\hline GCS at admission & - & - & 0.165 & - \\
\hline Brain edema using SEBES & - & - & 0.132 & - \\
\hline Predictors & aOR & $95 \% \mathrm{CI}$ & $p$ value & Points \\
\hline BNP serum level $>95.4 \mathrm{pg} / \mathrm{ml}$ & 5.7 & $1.782-18.761$ & $0.003^{*}$ & 1 \\
\hline Sodium serum level $<137.5$ & 4.6 & $1.460-14.469$ & $0.009^{*}$ & 1 \\
\hline SDH location (left side) & 4.4 & $1.265-15.044$ & $0.020^{*}$ & 1 \\
\hline Age & - & - & 0.171 & - \\
\hline Sex (female) & - & - & 0.131 & - \\
\hline Time until treatment & - & - & 0.411 & - \\
\hline Dementia & - & - & 0.284 & - \\
\hline Atrial fibrillation & - & - & 0.379 & - \\
\hline Operative technique (craniotomy) & - & - & 0.106 & - \\
\hline GCS at admission & - & - & 0.215 & - \\
\hline Brain edema using SEBES & - & - & 0.077 & - \\
\hline
\end{tabular}

$B N P$ brain natriuretic peptide, GCS Glasgow coma scale, $S D H$ subdural hematoma

* $p$ value significant

$(<137.5 \mathrm{mmol} / \mathrm{l})$, and left-sided aSDH could accurately predict the occurrence of EPS. Based on these variables, a prediction model was computed and was able to accurately predict EPS risk. Among patients with "intermediate" $($ score $=1)$ and "high" (score $=2 / 3$ ) risk for EPS,
$11.1 \%$ and $42.4 \%$ experienced EPS, respectively. In contrast, only $4.2 \%$ of the patients with "low risk" (score $=0$ ) developed EPS.

Jennett first described and reported the incidence of early PTS and EPS in traumatic aSDH and concluded that EPS differ from late 


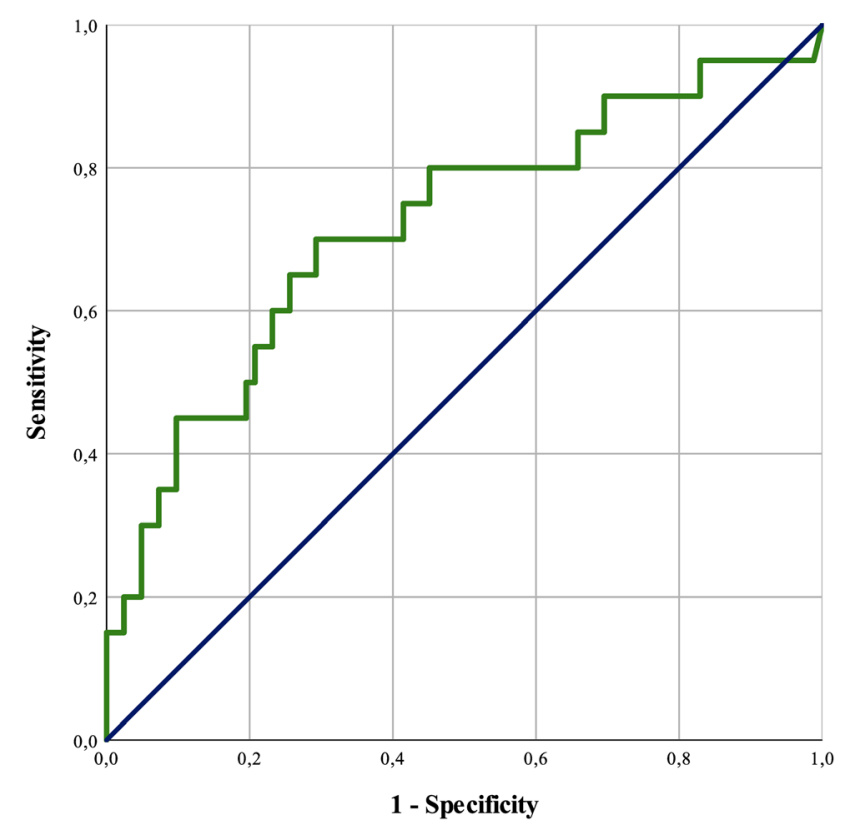

Fig. 2 Receiver-operating characteristics curve analysis for the prediction of early postoperative seizures considering BNP

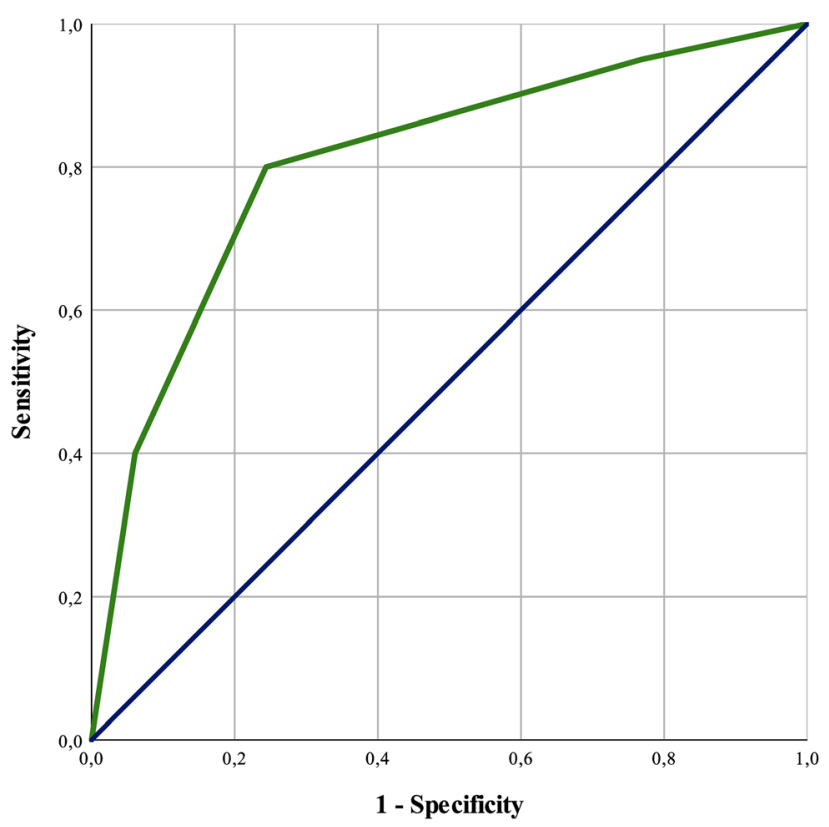

Fig. 3 Receiver-operating characteristics curve for the prediction of EPS considering the prediction model. The model showed in both cases an excellent discrimination according to Hosmer et al.

seizures that occurred in the subsequent weeks [17]. In critically ill patients, seizure activity can elevate the ICP, increase the lactate-to-pyruvate ratio, lengthen intensive care unit stay, and worsen long-term outcomes [28, 30, 40]. In particular, non-convulsive status epilepticus increases in-hospital mortality rate [34, 49] and the risk of unprovoked seizures [14]. Furthermore, the occurrence of EPS is an additional risk factor for the development of late seizures, 


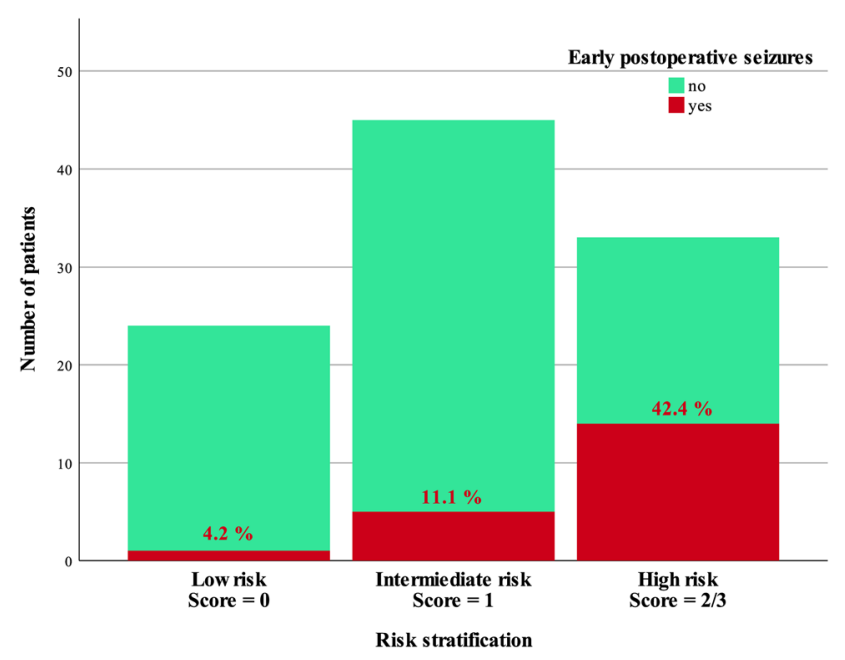

Fig. 4 Clustered bar chart depict the tendency of an increase of the risk to meet EPS with a higher score of the prediction model

Table 4 Increasing score of the prediction model shows an exponential increase in the risk of meeting early postoperative seizure using binomial logistic regression analysis (complete cohort)

\begin{tabular}{llll}
\hline Parameters & \multicolumn{2}{l}{ Early postoperative seizures $(\boldsymbol{N}=\mathbf{1 0 2})$} & $\boldsymbol{p}$ value \\
\cline { 2 - 4 } & $\mathbf{a O R}$ & $\mathbf{9 5 \%} \mathbf{C I}$ & - \\
\hline Score $=0$ (Reference) & - & - & 0.348 \\
Score $=1$ & 2.9 & $0.316-26.141$ & $0.009^{*}$ \\
Score $=2 / 3$ & 16.9 & $2.039-140.880$ & \\
\hline
\end{tabular}

${ }^{*} p$ value significant

leading to the diagnosis of epilepsy $[5,16-19,31]$. Up to $86 \%$ of patients with aSDH develop further seizures within 2 years after trauma [9]. Several pathophysiologic mechanisms have been postulated to explain the occurrence of EPS, including (1) irritation of the cortical surface of the brain with the hematoma itself or hematoma degradation compounds that are highly epileptogenic [7, 10], (in particular, hemoglobin degradation products) $[6,13]$; (2) sudden decompression through craniotomy that might cause an additional parenchymal injury through a sudden negative deceleration [32]; (3) intraoperative manipulation and traction of brain tissue [43]; (4) the formation of edema, hematoma, and pneumocephalus with subsequent brain injury through free-radical generation with an ensuing lack of cell membrane stability [12, 22-24].

Because of the paucity of research regarding EPS in the setting of traumatic aSDH, there is still a controversy concerning their clinical and prognostic values. Rabinstein et al. [30] showed that EPS are independently associated with worse functional outcome at discharge. However, regarding the last follow-up, the authors concluded that some patients may have achieved favorable recovery at a later time. But these results had a major limitation, as included patients had two different entities (acute and acute-on-chronic SDH) that were operated on using different operative techniques (craniotomy and burr hole trepanation), making the interpretation of the data difficult. Won et al. [43] performed the same study, but including 
only aSDH patients, and concluded that the postoperative occurrence of EPS or epileptiform discharges on EEG were significantly associated with poor functional outcome at discharge and follow-up. However, the study did not exclude patients with previous stroke or epilepsy, which makes these results not safe of probable biases. In contrast, in the study of Akbik et al. [2], in which only patients $\geq 65$ years were included, no significant relationship was found between EPS and functional outcomes. In our study, and in accordance with previous literature $[27,30,32,33,44]$, EPS were significantly associated with a postoperative poor neurologic condition, but not with poor functional outcome [2]. In the multivariate analysis, we found that a poor neurologic condition on the 7 th postoperative day could independently predict poor functional outcomes. Whether patients who experienced EPS can achieve a delayed functional recovery in the long-term requires further investigations.

Compared to chronic SDH, predictors of EPS in traumatic aSDH have been significantly under-studied, and specific data are still lacking. Here we examined several significant risk factors for seizures, including severe traumatic injury, decreased preoperative and postoperative GCS scores, timing of the operation, and AAT [3, 9, 10, 30, 38, 43, 47]. Interestingly, advanced age was not a predictor of EPS in our study, a finding that is in line with other recent study results $[30,43]$. In another study, patients with blood type A had increased risk for EPS, presumably through different clot properties and thereby higher cortex irritation [8]. In our cohort, EPS was more frequently observed in patients with blood type A (A: $60 \%$ vs. O: $20 \%$, B: $10 \%$, and $\mathrm{AB}: 10 \%)$, but not to a statistically significant extent. Furthermore, AAT has been previously reported as a predictor of EPS [43], but this was not the case in our study. This may be explained by differences in local policies regarding timing of operation $>24 \mathrm{~h}$ (5\% in our cohort vs. $23.7 \%$ [43]). In accordance with our findings, left-sided hematoma has also been identified as a predictor of EPS assuming that the clinical manifestation of left-sided seizures prompt clinical diagnosis to a greater extent than right-sided ones [30, 42]. Sodium serum concentration is a major determinant of several biologic pathways in the central nervous system, and hypo-osmolar states result in brain edema and seizures [26]. In line with the literature results regarding the association between hyponatremia and seizures, our study results showed that lower sodium concentration on admission was an independent predictor of EPS. Moreover, based on the well-known relationship between ICP and BNP in patients with TBI [35], our results indicated that BNP was an independent predictor of EPS. Further prospective studies should try to elucidate the pathophysiologic background of this increase.

The clinical implications of these findings are crucial for the clinical course and outcome of patients with traumatic aSDH at high risk of developing EPS. Although clinical guidelines for TBI recommend the use of antiepileptic drugs (AEDs) within the first 7 days after trauma in case of a GCS score $<10$ and/or abnormal cranial computer tomography (i.e., intracranial hematoma and/or brain contusion and/or depressed skull fracture) and/or when seizures accompany the presentation (Class I evidence, Level A recommendation for use of phenytoin in adult patients with severe TBI by the American Academy of Neurology) [6, 36], practice regarding prophylactic AEDs in traumatic aSDH remains controversial. Indeed, to date, there have been no randomized placebo-controlled studies investigating the values of prophylactic AEDs, specifically in the context of traumatic aSDH. Only one recently published retrospective study [31] compared the prophylactic use of levetiracetam vs. phenytoin in patients with traumatic aSDH, showing equal efficacy for the two agents but a significantly lower adverse event profile and better patient adherence with levetiracetam. However, no control group was assessed in that study. In TBI patients, late PTS were not significantly reduced after the use of prophylactic AEDs [13, 37, 48]. Besides, only one study could observe a delayed functional recovery of patients with early PTS after using phenytoin [44]. In aSDH patients, large-scale trials examining the optimal duration of seizure prophylaxis are lacking, and whether this can reduce the risk of late PTS remains unknown. Nonetheless, EPS remains a leading cause of 
postoperative morbidity in the neurologic intensive care unit, and it is critical to identify seizures expediently and treat them aggressively [7]. Following the recommendations of the American Academy of Neurology [6], some authors suggested using AEDs prophylactically within the first week after head trauma in patients with severe aSDH [43, 44]. Consequently, a prediction model would be helpful in clinical practice to identify patients at high risk that might benefit from the prophylactic use of AEDs. Our prediction model could accurately predict EPS, with an excellent discriminative capacity (AUC 0.81, 95\%CI 0.69-0.92), sensitivity of $70 \%$, specificity of $76.8 \%$, and a high negative predictive value $(87.1 \%)$, allowing a score of 0 to nearly rule out the occurrence of EPS. Furthermore, it allows a clear stratification of EPS risk and guides neurosurgeons and neurologists when counseling patients' relatives on the expected early neurologic and functional outcome at discharge. Nonetheless, this prediction model needs external validation. Also, further randomized placebo-controlled studies are required to clarify the ongoing debate regarding the advantage of using AEDs prophylactically in traumatic aSDH.

\section{Study Limitations}

Our study cohort does not represent the whole spectrum of patients with traumatic aSDH; instead, it focused only on surgically treated patients in good renal and cardiac health. This represents $73.9 \%$ of all individuals who needed and received surgical treatment and may limit the generalizability of our findings. Furthermore, the lack of postoperative EEG monitoring in this study could explain the lower rate of EPS compared to other studies $[30,43]$ in which all subclinical seizures could be assessed. Larger prospective studies should be conducted in different populations and settings with EEG monitoring to assess the "real" incidence of EPS.

\section{CONCLUSIONS}

Our findings demonstrate that EPS are a common complication after surgical evacuation of traumatic aSDH and seem to play a central role in the early postoperative phase, deteriorating patients' neurologic condition, leading to a poor functional outcome at discharge. Our study presents BNP as a novel reliable predictor of EPS. This biomarker may help both neurosurgeons and neurologists in further evaluation of EPS risk after surgery for traumatic aSDH. Further larger prospective studies are required to validate our findings and maybe reach a more precise estimate of EPS risk through BNP levels.

\section{ACKNOWLEDGEMENTS}

We thank the participants and their relatives for giving their consent to perform this prospective observational study which allowed giving a first interesting insight into the role of brain natriuretic peptide in the setting of traumatic acute subdural hematoma. Also, we thank the medical student Mr. Jan Rodemerk for his unconditional support in editing and processing our figures to a higher quality.

Funding. We acknowledge support by the Open Access Publication Fund of the University of Duisburg-Essen, which helped the authors to fund the journal's Rapid Service Fee.

Authorship. All named authors meet the International Committee of Medical Journal Editors (ICMJE) criteria for authorship for this article, take responsibility for the integrity of the work as a whole, and have given their approval for this version to be published.

Authors' Contributions. Dr. Chihi conceptualized and designed the study, acquired, analyzed, and interpreted the data, performed the statistical analyses, drafted the initial manuscript, and reviewed and revised the final version of the manuscript. Dr. Darkwah Oppong analyzed and interpreted the data and reviewed and revised the final version of the manuscript. Dr. Quesada analyzed and interpreted the data, controlled the statistical analyses, revised the manuscript for important intellectual content, and reviewed and revised the final version of 
the manuscript. Drs. Dinger and Gembruch helped acquiring the data, analyzed and interpreted the data, and reviewed the final version of the manuscript. Drs. Pierscianek, Ahmadipour, Uerschels, and Wrede revised the manuscript for important intellectual content and reviewed the final version of the manuscript. Prof. Sure supervised the study, revised the manuscript for important intellectual content, and reviewed the final version of the manuscript. Dr. Jabbarli analyzed and interpreted the data, supervised the study and the statistical analyses, revised the manuscript for important intellectual content, and reviewed the final version of the manuscript.

Disclosures. Mehdi Chihi, Marvin Darkwah Oppong, Carlos Martinez Quesada, ThiemoFlorin Dinger, Oliver Gembruch, Daniela Pierscianek, Yahya Ahmadipour, Anne-Kathrin Uerschels, Karsten Henning Wrede, Ulrich Sure, and Ramazan Jabbarli have no financial relationships relevant to this article to disclose and no conflicts of interest.

Compliance with Ethics Guidelines. This study was performed in accordance with the ethical standards of the institutional research committee and the Code of Ethics of the World Medical Association (Declaration of Helsinki). The Institutional Review Board approved the study protocol (Medical Faculty, University of Duisburg-Essen, Registration number: 15-6632BO). Written informed consent was obtained from all participants or their next of kin in case of altered state of consciousness.

Data Availability. The data that support the findings of this study are available in the Supplementary Material, and further data can be available on request from the corresponding author. Further data are not publicly available due to privacy restrictions.

Open Access. This article is licensed under a Creative Commons Attribution-NonCommercial 4.0 International License, which permits any non-commercial use, sharing, adaptation, distribution and reproduction in any medium or format, as long as you give appropriate credit to the original author(s) and the source, provide a link to the Creative Commons licence, and indicate if changes were made. The images or other third party material in this article are included in the article's Creative Commons licence, unless indicated otherwise in a credit line to the material. If material is not included in the article's Creative Commons licence and your intended use is not permitted by statutory regulation or exceeds the permitted use, you will need to obtain permission directly from the copyright holder. To view a copy of this licence, visit http://creativecommons.org/licenses/by$\mathrm{nc} / 4.0 /$.

\section{REFERENCES}

1. Abdelalim EM, Takada $\mathrm{T}$, Torii R, Tooyama I. Molecular cloning of BNP from heart and its immunohistochemical localization in the hypothalamus of monkey. Peptides. 2006;27: 1886-93.

2. Akbik OS, Starling RV, Gahramanov S, Zhu Y, Lewis J. Mortality and functional outcome in surgically evacuated acute subdural hematoma in elderly patients. World Neurosurg. 2019;126:e1235-41.

3. Annegers JF, Hauser WA, Coan SP, Rocca WA. A population-based study of seizures after traumatic brain injuries. N Engl J Med. 1998;338:20-4.

4. Bruno A, Akinwuntan AE, Lin C, Close B, Davis K, Baute V, Aryal T, Brooks D, Hess DC, Switzer JA. Simplified modified rankin scale questionnaire: reproducibility over the telephone and validation with quality of life. Stroke. 2011;42:2276-9.

5. Caveness WF, Portera A, Scheffner D. Epilepsy, a product of trauma in our time. Epilepsia. 1976;17: 207-15.

6. Chang BS, Lowenstein DH. Practice parameter: antiepileptic drug prophylaxis in severe traumatic brain injury: report of the Quality Standards Subcommittee of the American Academy of Neurology. Neurology. 2003;60:10-6.

7. Driver J, DiRisio AC, Mitchell H, Threlkeld ZD, Gormley WB. Non-electrographic seizures due to subdural hematoma: a case series and review of the literature. Neurocrit Care. 2019;30:16-21.

8. Dubinski D, Won S-Y, Behmanesh B, Brawanski N, Geisen C, Seifert V, Senft C, Konczalla J. The clinical 
relevance of $\mathrm{ABO}$ blood type in 100 patients with acute subdural hematoma. PLoS ONE. 2018;13: e0204331.

9. Englander J, Bushnik T, Duong TT, Cifu DX, Zafonte R, Wright J, Hughes R, Bergman W. Analyzing risk factors for late posttraumatic seizures: a prospective, multicenter investigation. Arch Phys Med Rehabil. 2003;84:365-73.

10. Frey LC. Epidemiology of posttraumatic epilepsy: a critical review. Epilepsia. 2003;44:11-7.

11. Frontera JA, Egorova N, Moskowitz AJ. National trend in prevalence, cost, and discharge disposition after subdural hematoma from 1998-2007. Crit Care Med. 2011;39:1619-25.

12. Fugate JE. Complications of neurosurgery. Continuum. 2015;21:1425-44.

13. Haltiner AM, Temkin NR, Dikmen SS. Risk of seizure recurrence after the first late posttraumatic seizure. Arch Phys Med Rehabil. 1997;78:835-40.

14. Hesdorffer DC, Logroscino G, Cascino G, Annegers JF, Hauser WA. Risk of unprovoked seizure after acute symptomatic seizure: effect of status epilepticus. Ann Neurol. 1998;44:908-12.

15. Hosmer DW Jr, Lemeshow S, Sturdivant RX. Applied logistic regression. Hoboken: Wiley; 2013.

16. Jennett B (1975) Epilepsy after non-missile head injuries. Heinemann Medical

17. Jennett WB. Early traumatic epilepsy: definition and identity. The Lancet. 1969;293:1023-5.

18. Jennett WB. Predicting epilepsy after blunt head injury. BMJ. 1965;1:1215.

19. Jennett WB, Lewin W. Traumatic epilepsy after closed head injuries. J Neurol Neurosurg Psychiatry. 1960;23:295.

20. Karibe H, Hayashi T, Hirano T, Kameyama M, Nakagawa A, Tominaga T. Surgical management of traumatic acute subdural hematoma in adults: a review. Neurologia medico-chirurgica:cr. 2014;54(11):887-94.

21. Kirchhoff C, Stegmaier J, Bogner V, Buhmann S, Mussack T, Kreimeier U, Mutschler W, Biberthaler P. Intrathecal and systemic concentration of NTproBNP in patients with severe traumatic brain injury. J Neurotrauma. 2006;23:943-9.

22. Lee L, Ker J, Quah BL, Chou N, Choy D, Yeo TT. A retrospective analysis and review of an institution's experience with the complications of cranioplasty. Br J Neurosurg. 2013;27:629-35.
23. Manaka S, Ishijima B, Mayanagi Y. Postoperative seizures: epidemiology, pathology, and prophylaxis. Neurol Med Chir. 2003;43:589-600.

24. Nishizawa Y. Glutamate release and neuronal damage in ischemia. Life Sci. 2001;69:369-81.

25. Pandey KN. Biology of natriuretic peptides and their receptors. Peptides. 2005;26:901-32.

26. Peri A. Morbidity and mortality of hyponatremia. Disord Fluid Electrolyte Metab. 2019;52:36-48.

27. Phuenpathom N, Choomuang M, Ratanalert S. Outcome and outcome prediction in acute subdural hematoma. Surg Neurol. 1993;40:22-5.

28. Pollandt S, Ouyang B, Bleck TP, Busl KM. Seizures and epileptiform discharges in patients with acute subdural hematoma. J Clin Neurophysiol. 2017;34: $55-60$.

29. Porzionato A, Macchi V, Rucinski M, Malendowicz LK, De Caro R. Natriuretic peptides in the regulation of the hypothalamic-pituitary-adrenal axis. In: International review of cell and molecular biology. Amsterdam: Elsevier; 2010. p. 1-39.

30. Rabinstein AA, Chung SY, Rudzinski LA, Lanzino G. Seizures after evacuation of subdural hematomas: incidence, risk factors, and functional impact. J Neurosurg. 2010;112:455-60.

31. Radic JAE, Chou SH-Y, Du R, Lee JW. Levetiracetam versus phenytoin: a comparison of efficacy of seizure prophylaxis and adverse event risk following acute or subacute subdural hematoma diagnosis. Neurocrit Care. 2014;21:228-37.

32. Seifi A, Asadi-Pooya AA, Carr K, Maltenfort M, Emami M, Bell R, Moussouttas M, Yazbeck M, Rincon F. The epidemiology, risk factors, and impact on hospital mortality of status epilepticus after subdural hematoma in the United States. Springerplus. $2014 ; 3: 332$.

33. Servadei F. Prognostic factors in severely head injured adult patients with acute subdural haematoma's. Acta Neurochir. 1997;139:279-85.

34. Shneker BF, Fountain NB. Assessment of acute morbidity and mortality in nonconvulsive status epilepticus. Neurology. 2003;61:1066-73.

35. Sviri G, Soustiel J, Zaaroor M. Alteration in brain natriuretic peptide (BNP) plasma concentration following severe traumatic brain injury. Acta Neurochir. 2006;148:529-33.

36. Swadron SP, LeRoux P, Smith WS, Weingart SD. Emergency neurological life support: traumatic brain injury. Neurocrit Care. 2012;17:112-21. 
37. Temkin NR. Antiepileptogenesis and seizure prevention trials with antiepileptic drugs: meta-analysis of controlled trials. Epilepsia. 2001;42:515-24.

38. Temkin NR. Risk factors for posttraumatic seizures in adults. Epilepsia. 2003;44:18-20.

39. Torbic H, Forni AA, Anger KE, Degrado JR, Greenwood BC. Use of antiepileptics for seizure prophylaxis after traumatic brain injury. Am J Health Syst Pharm. 2013;70:759-66.

40. Vespa PM, Miller C, McArthur D, Eliseo M, Etchepare M, Hirt D, Glenn TC, Martin N, Hovda D. Nonconvulsive electrographic seizures after traumatic brain injury result in a delayed, prolonged increase in intracranial pressure and metabolic crisis. Crit Care Med. 2007;35:2830.

41. Weimer JM, Gordon E, Frontera JA. Predictors of functional outcome after subdural hematoma: a prospective study. Neurocrit Care. 2017;26:70-9.

42. Won S-Y, Dubinski D, Brawanski N, Strzelczyk A, Seifert V, Freiman TM, Konczalla J. Significant increase in acute subdural hematoma in octo-and nonagenarians: surgical treatment, functional outcome, and predictors in this patient cohort. Neurosurg Focus. 2017;43:E10.

43. Won S-Y, Dubinski D, Herrmann E, Cuca C, Strzelczyk A, Seifert V, Konczalla J, Freiman TM. Epileptic seizures in patients following surgical treatment of acute subdural hematoma-incidence, risk factors, patient outcome, and development of new scoring system for prophylactic antiepileptic treatment (GATE-24 score). World Neurosurg. 2017;101: 416-24.

44. Won S-Y, Konczalla J, Dubinski D, Cattani A, Cuca C, Seifert V, Rosenow F, Strzelczyk A, Freiman TM. A systematic review of epileptic seizures in adults with subdural haematomas. Seizure. 2017;45: 28-35.

45. Wu X, Sha H, Sun Y, Gao L, Liu H, Yuan Q, Zhang T, Zhu J, Zhou L, Hu J. N-terminal pro-B-type natriuretic peptide in patients with isolated traumatic brain injury: a prospective cohort study. J Trauma Acute Care Surg. 2011;71:820-5.

46. Yablon SA. Posttraumatic seizures. Arch Phys Med Rehabil. 1993;74:983-1001.

47. Yeh C-C, Chen T-L, Hu C-J, Chiu W-T, Liao C-C. Risk of epilepsy after traumatic brain injury: a retrospective population-based cohort study. J Neurol Neurosurg Psychiatry. 2013;84:441-5.

48. Young B, Rapp RP, Norton J, Haack D, Tibbs PA, Bean JR. Failure of prophylactically administered phenytoin to prevent late posttraumatic seizures. J Neurosurg. 1983;58:236-41.

49. Young BG, Jordan KG, Doig GS. An assessment of nonconvulsive seizures in the intensive care unit using continuous EEG monitoring: an investigation of variables associated with mortality. Neurology. 1996;47:83-9. 